Available online on 15.03.2020 at http://jddtonline.info
Open Access to Pharmaceutical and Medical Research
unrestricted non-commercial use, provided the original work is properly cited

Open Access

Research Article

\title{
Preparation and Evaluation of Sodium Alginate Microparticles using Pepsin
}

\author{
Sankha Bhattacharya*, Vishal Puri, Shubham Sharma, Debasish Sahoo, Pradeep Kumar Pal, Sima Kujur, \\ Gaurav Goyal, Amandeep Singh
}

Department of Pharmaceutics, ISF College of Pharmacy, GT Road (NH-95), Ghal Kalan, Moga, Punjab 142001, INDIA

\begin{abstract}
Aim: The main aim of this article is to prepare and evaluate sodium alginate microparticles and evaluate on the basis of their characterization. The drug is dissolved, encapsulated or attached to a microparticles matrix. Depending upon method of preparation microparticles were obtained. Microparticles were developed as a carrier for vaccines and other disease like rheumatoid arthritis, cancer etc. Microparticles were developed to increase the efficacy of active pharmaceutical ingredient to a specific targeted site.

Material and Method: Microparticles of Sodium Alginate, Pepsin and Calcium Chloride were prepared in six batches (A-F) with different ratio of sodium alginate and calcium chloride respectively i.e. (0.25:2.5), (0.25:5), (0.25:7.5), (0.5:2.5), (0.5:5), (0.5:7.5) by using a homogenizing method. Microparticles were evaluated for particle size distribution, zeta potential and morphology.

Result and Discussion: The normal particle size of each of the six batches were analyzed by Zeta Sizer (Delsa C Particle Analyzer) and it was found that the Batch B (0.25:5) delivered the best microparticles with size distribution of 1.2731 ( $\mu \mathrm{m}$ ). All batches were seen under Motic magnifying microscope by using the Sulforhodamine B (M.W. 479.02) color as staining dye. Microparticles was found to be semi spherical in shape.

Conclusion: Results of all the six batches was contrasted based on particle size investigation, zeta potential and morphology. Batch B (0.25:5) was considered as the best formulation.
\end{abstract}

Key words: Micro Particle, Pepsin, Sodium Alginate and Calcium Chloride, Sulforhodamine B, Zeta Sizer.

Article Info: Received 02 Jan 2020; Review Completed 05 Feb 2020; Accepted 14 Feb 2020; Available online 15 March 2020

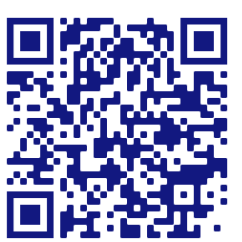

\section{Cite this article as:}

Bhattacharya S, Puri V, Sharma S, Sahoo D, Pal PK, Kujur S, Goyal G, Singh A, Preparation and Evaluation of Sodium Alginate Microparticles using Pepsin, Journal of Drug Delivery and Therapeutics. 2020; 10(2):5-11 http://dx.doi.org/10.22270/jddt.v10i2.3901

Dr. Sankha Bhattacharya, Associate Professor, Department of Pharmaceutics, ISF College of Pharmacy, GT Road (NH-95), Ghal Kalan, Moga, Punjab 142001, INDIA

\section{INTRODUCTION}

Microparticles are defined as a solid dispersion with a size range between 1 to $1000 \mu \mathrm{m}$ in diameter[1]. Microparticles can be directly injected into the target tissues without damaging the specific tissue. Blood Brain Barrier (BBB) protects the central nervous system against toxins and makes it difficult for a drug to reach the brain to treat certain disease[2]. Microparticles help in minimize the toxic effect and loss of drug in non-targeted sites. Drug-loaded biodegradable microparticles can be directly injected into the brain tissue to improve the efficiency of drug to treat certain disease[3]. Microparticles have a high surface area for a drug to release and have a short diffusion pathway that helps to increase the therapeutic efficacy and reduces the side effects[4]. Biodegradable polymeric microparticles are coated with a hydrophilic polymer such as polyethylene glycol (PEG) which are used in a drug delivery device because of their ability to bind with a particular organ for a prolonged period of time[5]. PEG act as a carrier of DNA in a gene therapy and their ability to deliver proteins, peptides and genes to a particular part of a human body[6]. When microparticles are administered in the form of drug into the stomach or GIT tract then drug get absorbed into the blood and distributed throughout the system. When the drug is absorbed into the blood then there is no uniform delivery of drug to a target site which results in decrease in concentration[7]. To avoid low concentration of drug in the blood, controlled or sustained drug delivery system is preferred which helps the drug to incorporate within a polymeric matrix that controls the release rate and results in constant drug concentration at site of action for a prolonged period of time[8]. 


\section{Advantages}

1) Microparticles helps in enhancing solubility of poorly water-soluble drugs.

2) They possess immediate release properties and can give $80 \%$ or more of the drug in less time.

3) They help in targeted release of active pharmaceutical ingredient and prolong the drug release.

4) They help in increasing the bioavailability of drugs.

5) They help in minimize or eliminate side effects.

6) They help in reducing the dose size and dosing frequency which help to maintain the plasma concentration and improve patience compliance.

7) Size optimization and use of various targeting ligands can be conjugated with a drug to achieve a controlled/sustained release of drug to a specific targeted site.

8) They can act as a carrier for drugs and vaccines in diagnostic and surgical procedures.

9) Microparticles can be used for various routes of administration like oral, nasal, parentral, intraocular etc.

10) There will be a less chances of dose dumping and ensure better compatibility.

\section{Limitation}

1) Small particle size and large surface area result in limited drug loading and particle aggregation.

2) Microparticles have less reproducibility.

3) Different process like change in temperature, $\mathrm{pH}$ and solvent addition helps in achieving the stability of core particles.

Pepsin is produced in the stomach and is one of the main digestive enzymes where it digests the proteins in food inside the digestive system in humans. Pepsin is most active in acidic medium between $37^{\circ} \mathrm{C}$ and $42^{\circ} \mathrm{C}$. Pepsin show maximum activity at $\mathrm{PH}-2$ and minimum activity at $\mathrm{PH}-$ 6.5[9]. Sodium Alginate (ALG) is a hydrophilic polymer which is obtained from seaweeds. ALG is a biocompatible, non-irritant polymer which has swelling, gelling, mucoadhesive and stabilizing properties[10]. Sodium Alginate is also used as thickening agent in food industry. Cross linking of ALG with calcium chloride decrease the swelling ratio and enhance mucoadhesive property of the drug[11]. This is a prominent approach of obtaining microparticles and modified release in drug delivery system. Sodium Alginate is able to form a gel when dissolved in water in the presence of a divalent cation ( $\left.\mathrm{Ca}^{2+i o n s}\right)$ when cross linked with calcium chloride or exposed to certain salts[12]. This cross-linking reaction between sodium alginate and calcium chloride forms a spherical or semi spherical beads. Pepsin is also obtained from the gastric mucosa of pigs, cattle and sheep. So, binding of pepsin with sodium alginate will helps in enhancing the mucoadhesive property in gastric mucosa[13].

\section{MATERIALS AND METHODS}

The analytical grade of each substance was used in these experiments. Sodium Alginate (Algin, a cream-colored pale yellowish-brown powder, soluble in water forming a viscous colloidal solution, LOD-15.0\% at 105을 for $4 \mathrm{hrs}$.) and pepsin (from the porcine stomach, 1:3000) were purchased from central drug house (p) LTD. New Delhi-110002.Calcium chloride $\left(\mathrm{CaCl}_{2} 2 \mathrm{H}_{2} \mathrm{O}\right.$, M.W-147.02) was supplied from Loba Chemie Pvt. Ltd. Wodehouse Road, Mumbai, 400005, India.

\section{Method of Preparation}

Sodium Alginate solution was prepared in different concentration i.e. $0.25 \%$ and $0.5 \%$. Sodium Alginate solution was prepared by dissolving sodium alginate in distilled water and solution was stirred continuously with slightly heating at $55^{\circ} \mathrm{C}-60^{\circ} \mathrm{C}$ to dissolve sodium alginate in water. After cooling the sodium alginate solution, $0.6 \mathrm{mg}$ of pepsin was added and the drug loaded sodium alginate was prepared. The calcium chloride solution was also prepared in different concentrations. i.e. $2.5 \%, 5 \%, 7.5 \%$. The drug loaded sodium alginate solution was dropwise added into the calcium chloride solution by putting the calcium chloride solution in a magnetic stirrer (made by Remi equipment) and stirred at $1000 \mathrm{rpm}$. By adding different concentrations solution of sodium alginate with different concentrations of calcium chloride solution, a total of 6 different formulations were developed. After that, all the 6 solutions were homogenized by a homogenizer mixer made by Remi motor at $1250 \mathrm{rpm}$. Then micron size of sodium alginate microparticles were formed by continuous stirring for 15-20 minutes. The size of particles of 6 different solutions was evaluated by using dynamic light scattering zeta sizer (made by Delsa nano C particle Analyzer). Zeta potential can also be checked for different samples by using the same dynamic light scattering zeta sizer. (Figure 1\& 2).

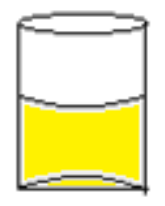

Sodium alginate solution
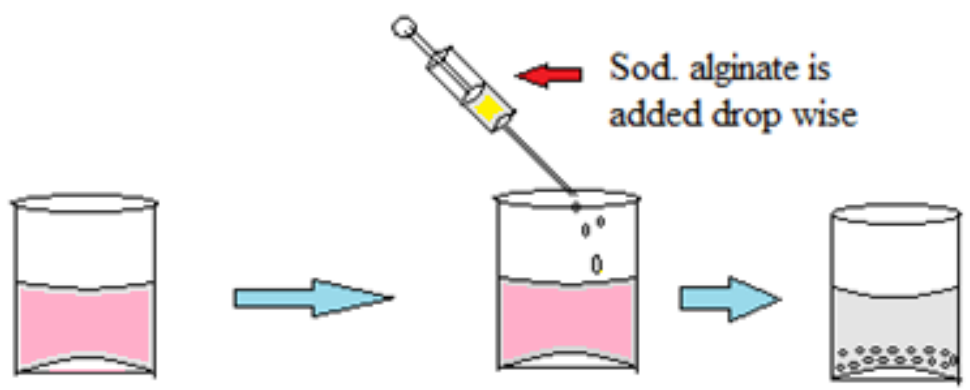

Calcium chloride solution

Microparticles

Figure 1 Diagrammatic representation of experiment 


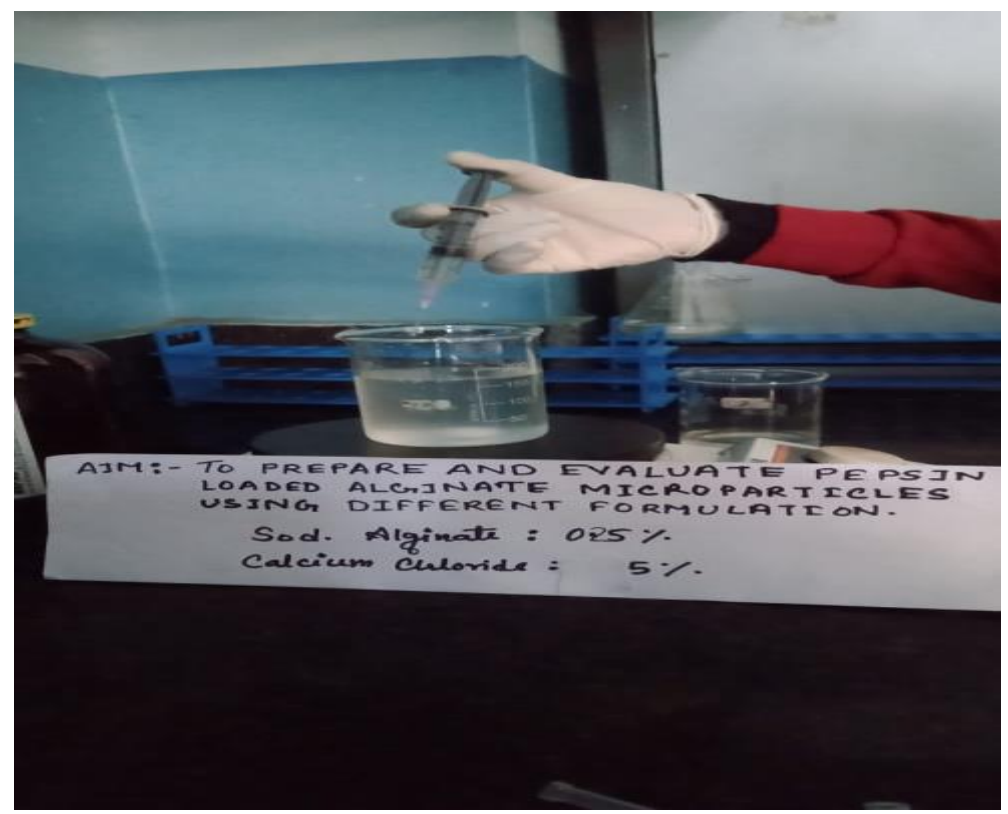

Figure 2: Dropwise addition of Sodium Alginate into Calcium Chloride Solution using Magnetic Stirring

\section{Evaluation of Microparticles}

\subsection{Standard Curve of Pepsin}

To get concentration of $0.6 \mathrm{mg}$, dilution was made from stock solution of Pepsin $(100 \mu \mathrm{g} / \mathrm{ml})$. Absorbance of Pepsin at $275 \mathrm{~nm}$ was taken in UV/Visible Spectrometer. The calibration curve was plotted between Absorbance verses Concentration. (Figure 3).

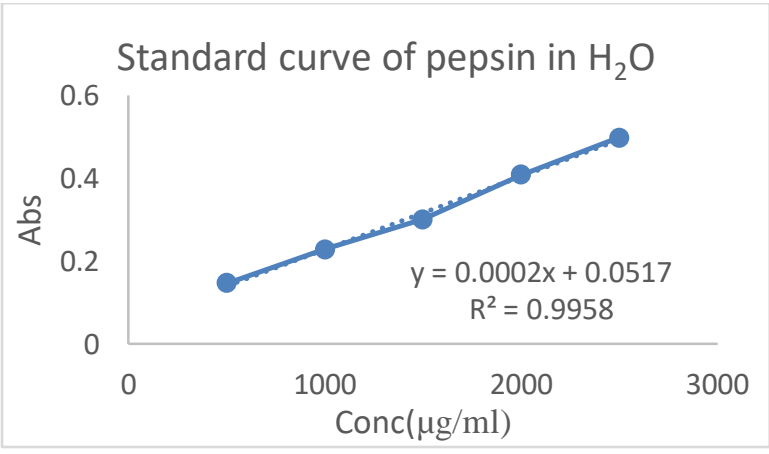

Figure 3: Standard Curve of Pepsin

\subsection{Determination of Drug Content}

For determination of drug content, $10 \mathrm{mg}$ of beads were placed in $100 \mathrm{ml}$ of distilled water for 24 hours. The solution was than filtered and measured for the drug content using UV Spectrophotometer. Drug content was calculated using a standard calibrated curve. The drug loading capacity was calculated using a following equation:

$$
\text { Drug Loading }(\%)=\frac{\text { Total amount of drug in particle }}{\text { Weight of particle taken }} \times 100
$$

Drug loading can be done at the time of microparticles production by incubating the carrier with a concentrated drug solution.

\subsection{Scanning Electron Microscopy (SEM)}

Scanning electron microscopy is a type of electron microscope that scan the surfaces with a focused beam of electrons to produce images. SEM was mainly used to examine the morphology of the sample. The sample is prepared by mounting the beads on the stub. The stub was coated either with gold or carbon. The gel beads were then observed under a scanning electron microscope.

\subsection{Particle Size Distribution}

Particle size determination was carried out using an optical microscope. A suspension of sodium alginate beads containing pepsin was prepared in a beaker. A droplet of pepsin loaded sodium alginate microparticles were suspended on a glass slide. A cover slip was gently placed on the sample. While placing the cover slip, no bubbles should be entrapped inside the cover slip. The average diameter of beads was calculated by following equation

$$
\mathrm{X}=\frac{\Sigma(\mathrm{Xi})}{\mathrm{N}}
$$

$\mathrm{X}=$ Average Particle Diameter,

$\mathrm{X}_{\mathrm{i}}=$ Individual diameter of beads

$\mathrm{N}=$ Number of Beads

\subsection{Particle Size and Zeta Potential Analysis}

Measurement of particle size and Zeta Potential was done by Dynamic Light Scattering (Delsa Nano C particle Analyzer)[14]. For particle size measurement, size cell or disposable rectangular cell was used whereas for Zeta Potential measurement, flow cell or high concentration cell was used. The lyophilized microparticles were placed in a standard glass cuvette. The glass cuvette was slowly filled with homogenized sample by syringe. No bubbles should be entrapped inside the cuvette. After opening the lid sample was placed and analyzed.

\subsection{Morphology}

\subsubsection{Microscopy Observation and Particle Size}

Morphology of pepsin loaded sodium alginate microparticles were determined by using Motic Microscope. A droplet of pepsin loaded sodium alginate microparticles were suspended on a glass slide (3"x 1"x $1 \mathrm{~mm})$. Samples were stained with a Sulforhodamine B solution (prepared by using $0.5 \%$ solution of acetic acid and distilled water). A cover slip was gently placed on the sample. While placing the cover slip, no bubbles should be entrapped inside the cover slip. Sample placed on a glass slide was observed under microscope with a magnification of 40x.Under microscope it 
was assumed that particle size was found to be semi spherical in shape.

\subsection{Entrapment Efficiency}

Drug Entrapment Efficiency refers to a load of drug (pepsin) into a carrier (alginate microparticles). This entrapment efficiency was determined by using UV Spectroscopy Method. For this, $10 \mathrm{ml}$ of pepsin loaded alginate microparticles was loaded into Centrifugation tube at $1000 \mathrm{rpm}$ for $10 \mathrm{~min}$. Supernatant was analyzed by using UV/ Visible Spectrophotometer at $275 \mathrm{~nm}$. Drug concentration was determined using standard calibration curve.

Entrapment Efficiency was calculated using formul

$$
\text { Entrapment Efficiency (EE) \% }=\frac{C t-C f}{C t} \mathrm{X} 100
$$

Where,

$\mathrm{C}_{\mathrm{t}}$ is Concentration of Total Drug

$\mathrm{C}_{\mathrm{f}}$ is Concentration of Entrapped Drug

\subsection{In-vitro Drug Release Studies}

In-vitro drug release studies were performed by using USP Dissolution Type II apparatus (Paddle Type). The drug release studies were carried out by adding $900 \mathrm{ml}$ of phosphate buffer solution $(7.4 \mathrm{PH})$ into vessel and temperature was maintained at $37^{\circ} \mathrm{C} \pm 0.5^{\circ} \mathrm{C}$. The speed of stirrer was maintained at $50 \mathrm{rpm}$. Accurately weighed samples of sodium alginate beads were added into the vessels. $5 \mathrm{ml}$ of solution was withdrawn and $5 \mathrm{ml}$ of solution was added into the vessel after predetermined time interval (30 minutes) to maintain sink condition. The sample withdrawn is then filtered by Whatman filter paper and absorbance was analyzed using UV Spectrophotometer at $275 \mathrm{~nm}$. The experiment was performed in triplicate

\section{RESULT AND DISCUSSION:}

Microparticles were prepared by modified emulsification method. Sodium Alginate which is an anionic polysaccharide, drug is loaded into it. Sodium Alginate was dropped into calcium chloride solution with constant stirring through syringe. Monovalent sodium ion show reaction with divalent calcium ion, it replaces the sodium with calcium ions which results in the formation of calcium alginate microparticles.

\subsection{Standard Curve of Pepsin}

The calibration curve was plotted between absorbance verses concentration.

\subsection{Morphology Study}

The microparticles were observed under a Motic Microscope and the particle were observed semi spherical in shape. The images taken from the microscope are shown in Figure 4.

\subsection{Size Distribution Studies}

The size distribution studies was done using dynamic light scattering technology (Zeta Sizer). The size of the microparticles varies from Batch A to F. Lowest value was observed in Batch B i.e. $1273.1 \mu \mathrm{m}$ and Highest value was observed in Batch F i.e. $91.4 \mu \mathrm{m}$. (Figure 5)

\subsection{Zeta Potential Studies}

Zeta Potential of pepsin containing microparticles was determined by using Zeta Sizer. Zeta Potential was measured for few batches and values varies from -2.61 (Batch A) to 3.13 (Batch C) (Figure 6).
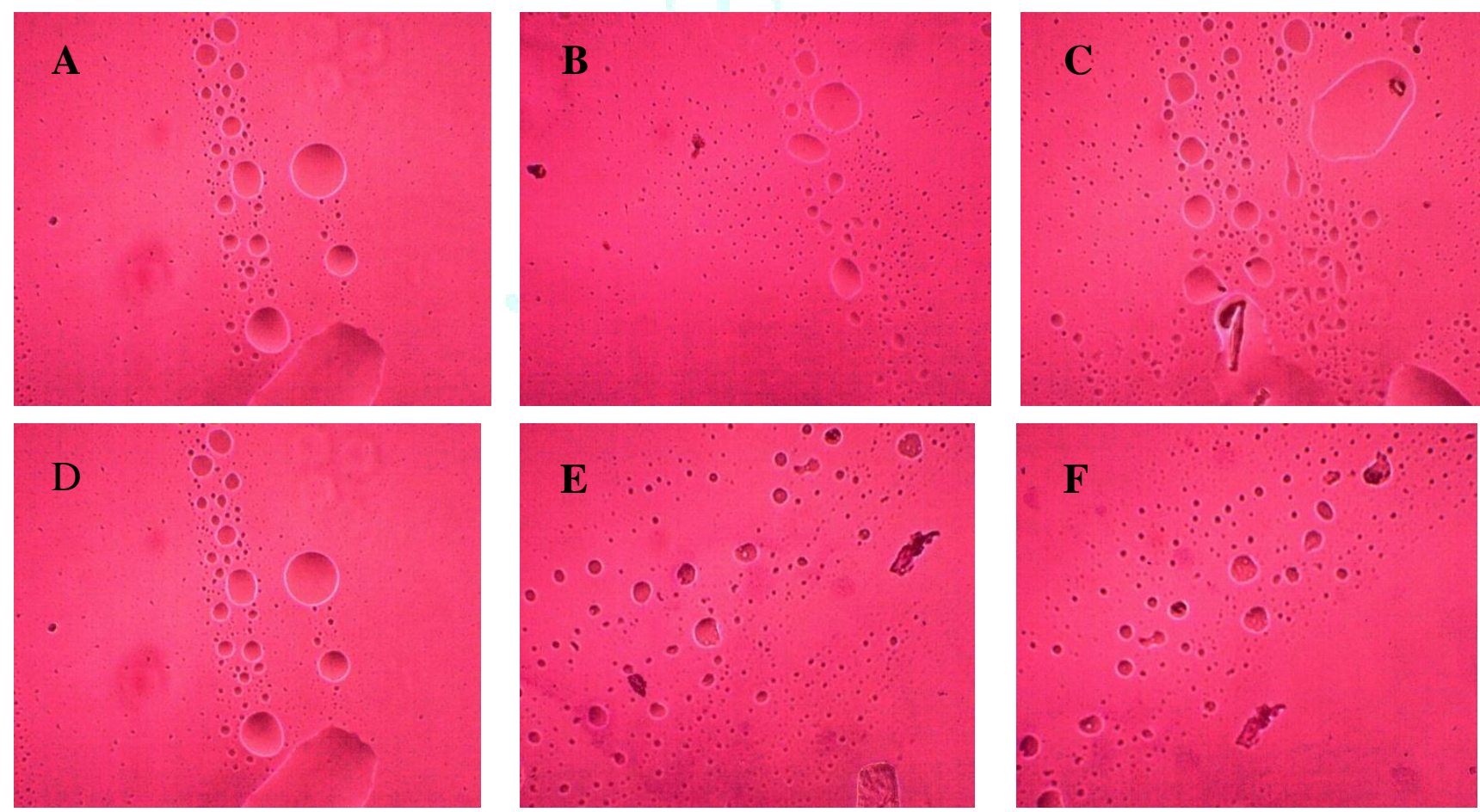

Figure 4 Morphological Studies of Microparticles containing Pepsin using motic microscope (A) Morphology of Pepsin containing Microparticles at $(0.25: 2.5)$ ratio of Sodium Alginate :Calcium Chloride (B)Morphology of Pepsin containing Microparticles at (0.25:5) ratio of Sodium Alginate :Calcium Chloride (C) Morphology of Pepsin containing Microparticles at (0.25:7.5) ratio of Sodium Alginate: Calcium Chloride (D)Morphology of Pepsin containing Microparticles at $(0.5: 2.5)$ ratio of Sodium Alginate: Calcium Chloride(E) Morphology of Pepsin containing Microparticles at (0.5:5) ratio of Sodium Alginate: Calcium Chloride (F) Morphology of Pepsin containing Microparticles at (0.5:7.5)ratio of Sodium Alginate: Calcium Chloride 


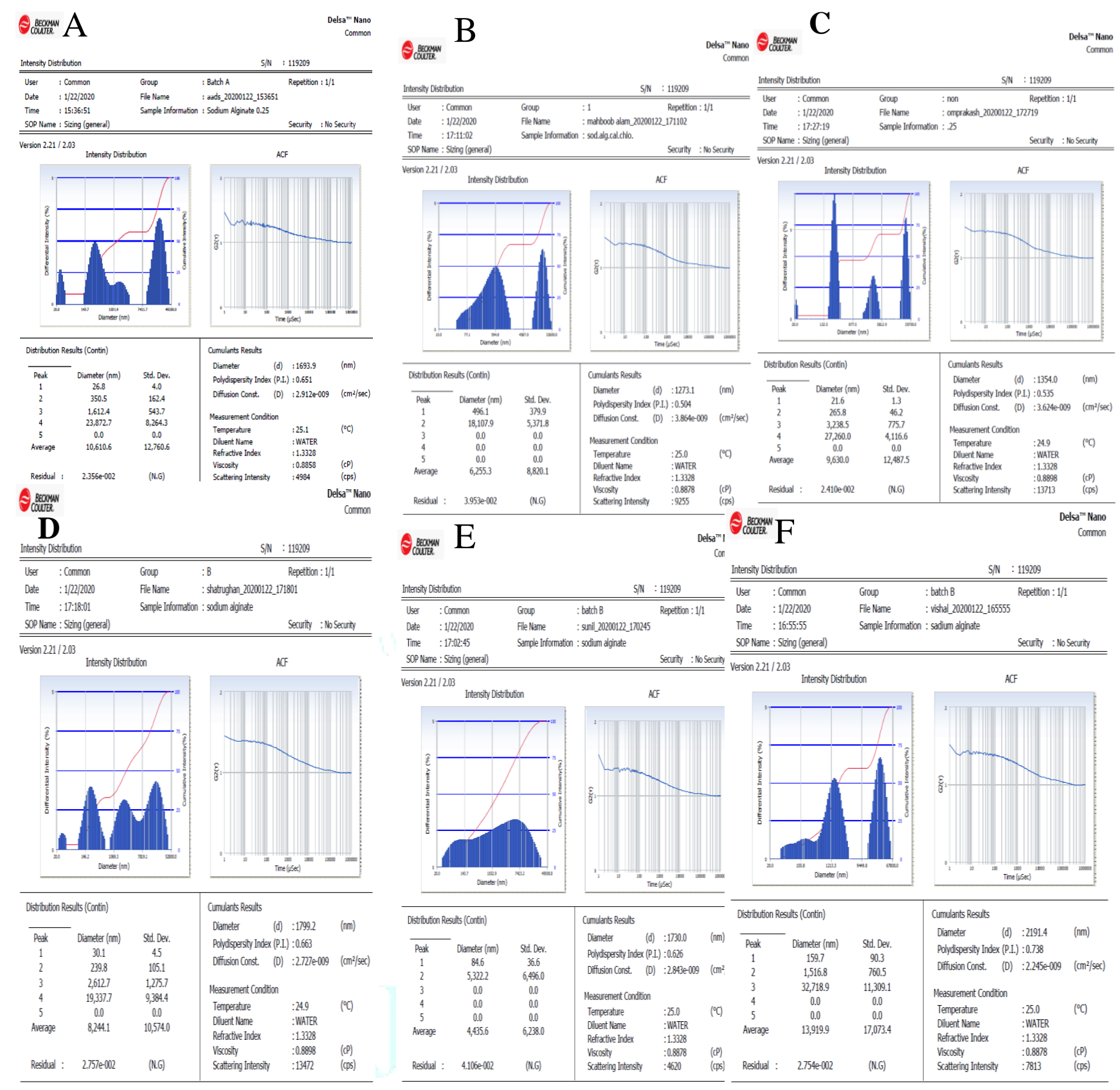

Figure5: Particle Size Analysis using Dynamic Light Scattering (A) Particle Size of Pepsin containing Microparticles at 0.25:2.5 ratio Sodium Alginate: Calcium Chloride $\quad$ (B) Particle Size of Pepsin containing Microparticles at 0.25:5 ratio Sodium Alginate: Calcium Chloride (C) Particle Size of Pepsin containing Microparticles at 0.25:7.5 ratio Sodium Alginate: Calcium Chloride (D) Particle Size of Pepsin containing Microparticles at 0.5:2.5 ratio Sodium Alginate: Calcium Chloride (E) Particle Size of Pepsin containing Microparticles at 0.5:5 ratio Sodium Alginate: Calcium Chloride (F) Particle Size of Pepsin containing Microparticles at 0.5:7.5 ratio Sodium Alginate: Calcium Chloride 


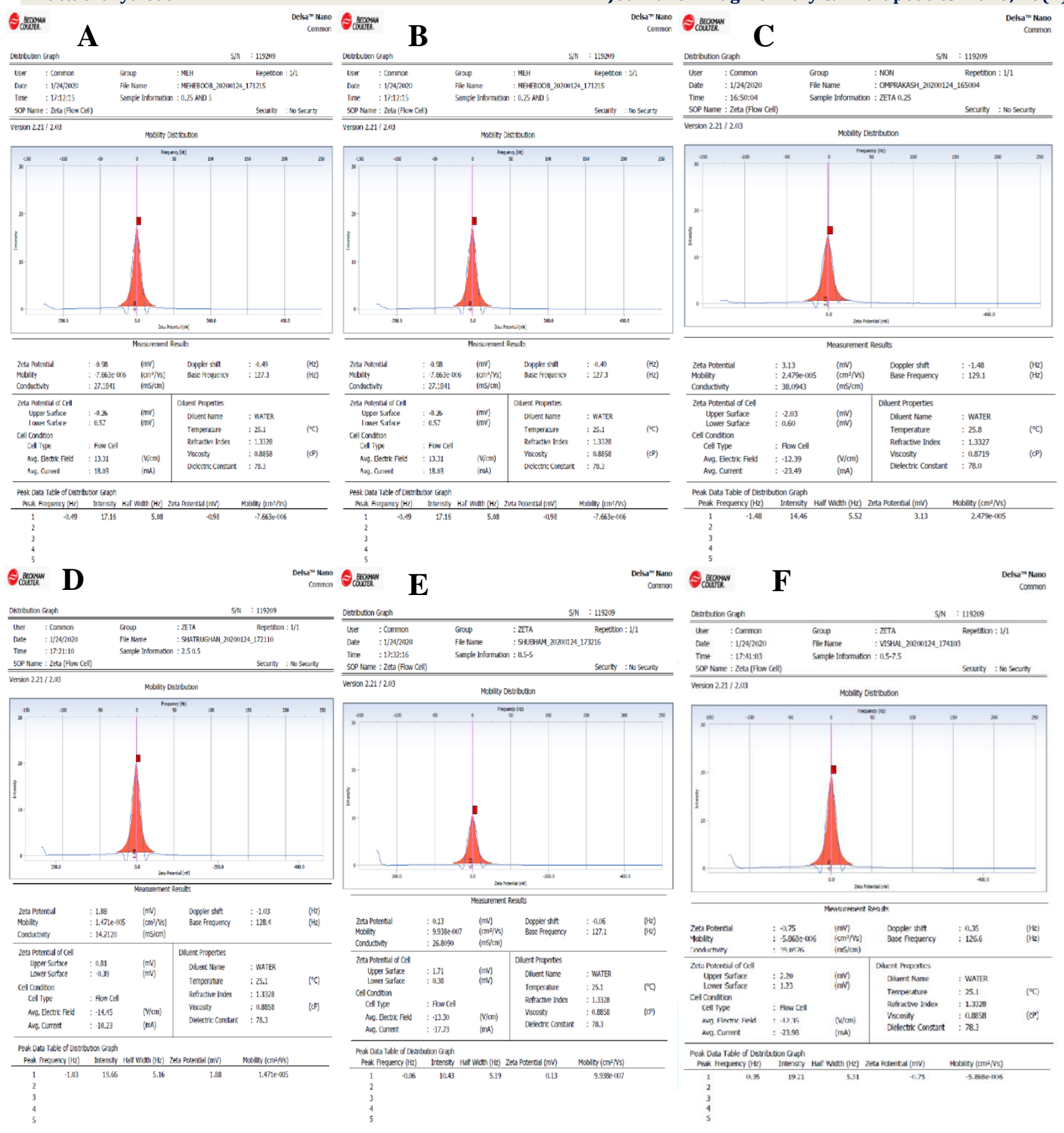

Figure 6:Zeta Potential is calculated by using Zeta Sizer (A) Zeta Potential of Pepsin containing Microparticles at 0.25:2.5 ratio of Sodium Alginate: Calcium Chloride (B) Zeta Potential of Pepsin containing Microparticles at 0.25:5 ratio of Sodium Alginate: Calcium Chloride (C) Zeta Potential of Pepsin containing Microparticles at 0.25:7.5 ratio of Sodium Alginate: Calcium Chloride (D) Zeta Potential of Pepsin containing Microparticles at 0.5:2.5 ratio of Sodium Alginate: Calcium Chloride (E) Zeta Potential of Pepsin containing Microparticles at 0.5:5 ratio of Sodium Alginate: Calcium Chloride (F) Zeta Potential of Pepsin containing Microparticles at 0.5:7.5 ratio of Sodium Alginate: Calcium Chloride

\section{CONCLUSION:}

The sodium alginate microparticles (polymer) were prepared at different ratio $0.25: 0.5$ and calcium chloride (crosslinking agent) was prepared at ratio 2.5:5:7.5. These formulations were loaded with pepsin. Different test was evaluated at 6 different concentrations.

\section{Acknowledgement}

Authors are thankful to ISF College of Pharmacy, Moga for providing facilities to carry out research and also thankful to respected Director Sir, Professors of ISF College of Pharmacy, Moga, Punjab. 


\section{REFERENCES:}

1. Huang J., et al., Nifedipine solid dispersion in microparticles of ammonio methacrylate copolymer and ethylcellulose binary blend for controlled drug delivery: Effect of drug loading on release kinetics. International journal of pharmaceutics, 2006; 319(1-2):44-54.

2. Garcia-Garcia E., et al., Colloidal carriers and blood-brain barrier (BBB) translocation: a way to deliver drugs to the brain? International journal of pharmaceutics, 2005; 298(2):274-292.

3. Parveen S. and S.K. Sahoo, Polymeric nanoparticles for cancer therapy. Journal of drug targeting, 2008; 16(2):;108-123.

4. Klose D., et al., How porosity and size affect the drug release mechanisms from PLGA-based microparticles. International journal of pharmaceutics, 2006; 314(2):198-206.

5. Soppimath K.S., et al., Biodegradable polymeric nanoparticles as drug delivery devices. Journal of controlled release, 2001; $70(1-2): 1-20$

6. Putnam D., Polymers for gene delivery across length scales. Nature materials, 2006; 5(6):439-451.

7. Ensign L.M., Cone R, and Hanes J, Oral drug delivery with polymeric nanoparticles: the gastrointestinal mucus barriers. Advanced drug delivery reviews, 2012; 64(6):557-570.
8. Santini J., John T, et al., Microchips as controlled drug-delivery devices. Angewandte Chemie International Edition, 2000; 39(14):2396-2407.

9. Lloyd A.L., Cloning, characterisation and sequencing of promoters of Helicobacter pylori 4187E. 2004: University of Western Australia.

10. Andrianov A.K. and Payne LG, Polymeric carriers for oral uptake of microparticulates. Advanced drug delivery reviews, 1998; 34(2-3):155-170.

11. Patil P., Chavanke D, and Wagh M, A review on ionotropic gelation method: novel approach for controlled gastroretentive gelispheres. Int J Pharm Pharm Sci, 2012; $4(4): 27-32$.

12. Sun J. and Tan $H$, Alginate-based biomaterials for regenerative medicine applications. Materials, 2013; 6(4):1285-1309.

13. Kumar M.R., et al., Chitosan chemistry and pharmaceutical perspectives. Chemical reviews, 2004; 104(12):6017-6084.

14. $\mathrm{Xu}$ R., Progress in nanoparticles characterization: Sizing and zeta potential measurement. Particuology, 2008; 6(2):112115. 